\title{
Espectroscopia óptica de baixo custo: uma estratégia para a introdução de conceitos de física quântica no ensino médio
}

\author{
Low cost optical spectroscopy: a strategy to introduce quantum physics concepts in high school
}

\author{
Aissa L. Azevedo ${ }^{1}$, Anderson K. S. Sousa ${ }^{1}$, Tiago J. Castro ${ }^{* 1 @}$ \\ ${ }^{1}$ Instituto Federal de Educação, Ciência e Tecnologia de Brasília, DF, Brasil
}

Recebido em 17 de Dezembro, 2018. Revisado em 18 de Fevereiro, 2019. Aceito em 25 de Março, 2019

\begin{abstract}
Neste artigo, reportamos a confecção e discutimos a aplicabilidade de um espectrômetro óptico de baixo custo como recurso didático para a introdução de conceitos de Física Quântica no ensino médio. O protótipo foi desenvolvido usando grade de difração comercial, lentes convergentes (lupas), webcam e o software Tracker para extração dos dados. Espectros de emissão de fontes luminosas, como lâmpada fluorescente, LEDs e lasers, foram coletados e suas potencialidades para subsidiar a introdução dos conceitos de níveis eletrônicos, banda de energia proibida (band gap), emissão e absorção de fótons, etc., foram exploradas. Propomos também uma atividade experimental com base na fotoexcitação de LEDs para tratar da relação entre energia do fóton e seu comprimento de onda. Além disso, mostramos que o protótipo pode ser satisfatoriamente empregado para a obtenção de espectros de absorção, os quais podem ser usados como elemento motivador para o estudo da interação entre radiação e matéria. Por fim, à luz dos resultados encontrados, discutimos a aplicação da espectroscopia óptica como uma estratégia para atender às diretrizes dos Parâmetros Curriculares Nacionais brasileiros quanto à inserção da Física Quântica na educação básica.
\end{abstract}

Palavras-chave: Espectroscopia óptica; Experimentos de baixo-custo; ensino de Física

In this paper, we report the production and discuss the applicability of a low cost optical spectrometer as a didactic resource for the introduction of Quantum Physics concepts in high school. The prototype was developed using commercial diffraction grating, convergent lenses (magnifying glass), webcam and the software Tracker for data extraction. Emission spectra of light sources, such as fluorescent lamps, LEDs and lasers, were collected and their potentialities to support the introduction of the concepts of electronic levels, band gap energy, emission and absorption of photons, etc., were explored. We also propose an experimental activity based on LED photoexcitation to treat the relationship between photon energy and its wavelength. In addition, we showed that the prototype can be satisfactorily used to obtain absorption spectra, which can be used as a motivating element for the study of the interaction between radiation and matter. Finally, in the light of the results obtained, we discussed the application of optical spectroscopy as a strategy to reach the recommendations of the Brazilian National Curricular Parameters regarding the insertion of Quantum Physics in basic education.

Keywords: optical spectroscopy; low cost experiments; Physics teaching

\section{Introdução}

A espectroscopia é um ramo da Física que lida com o estudo da radiação absorvida, refletida, emitida ou espalhada por uma substância. Existem tantos tipos de espectroscopia como existem tipos de radiações. Do ponto de vista histórico, o início da espectroscopia data do século XVII, quando Isaac Newton observou que a luz do Sol continha todo o espectro visível. O termo "espectro" foi então empregado primeiramente por Newton nesta época [1]. No início do Século XIX, já se conhecia a existência da radiação infravermelha (IV) e ultravioleta (UV), de forma que o desenvolvimento de espectrômetros

*Endereço de correspondência: tiago.castro@ifb.edu.br ópticos durante a primeira metade deste período permitiu que numerosos espectros fossem registrados, tais como os das cores de chamas e os das radiações emitidas por gases submetidos a descargas elétricas. Estes conhecimentos possibilitaram o desenvolvimento da teoria atômica e o surgimento da Mecânica Quântica, a qual modificou os rumos da Física durante o Século XX.

Além de sua importância para o desenvolvimento de teorias da Física, os diversos ramos da espectroscopia desempenham atualmente um papel central em investigações científicas e tecnológicas, muitas das quais com foco em aplicações práticas. Técnicas espectroscópicas são usadas por exemplo para a determinação da concentração de constituintes em fármacos e alimentos. Além disso, são empregadas rotineiramente na perícia criminal 
para identificar substâncias presentes em cenas de crimes e na produção de novos materiais, os quais integram principalmente os dispositivos eletrônicos desenvolvidos hoje [2].

No entanto, apesar de sua importância, a espectroscopia ainda é pouco explorada para o ensino de Física. Devido à sua natureza interdisciplinar, esta técnica tem grandes potencialidades para integrar as áreas de Física, Química e Biologia. Além disso, favorece a inserção de atividades experimentais em ambientes formais de ensino [3]. Considerando seu custo elevado, não é plausível que instituições de educação básica adquiram um espectrômetro comercial. No entanto, a confecção de um espectrômetro óptico de baixo custo pode ser feita por professores e alunos de nível médio e seu reflexo sobre o aprendizado de temas mais complexos relacionados com as áreas de ciências pode trazer ganho inestimável.

Assim, apresentamos neste trabalho os resultados decorrentes da confecção de um espectrômetro óptico de baixo custo para a aplicação em atividades de ensino de Física. São tratados os detalhes da construção do protótipo, sua aplicação para o estudo da radiação produzida por lâmpadas, LEDs e lasers, bem como sua utilização para obtenção de espectro de absorção.

\section{Materiais e Métodos}

A Figura 1 mostra o protótipo desenvolvido neste trabalho. As partes principais do espectrômetro óptico foram montadas dentro de uma caixa de MDF (Medium-Density Fiberboard) a qual foi pintada com tinta preta. Em um dos lados da caixa foi feita uma fissura e com o auxílio de duas pequenas placas metálicas foi construída uma fenda de $\sim 0.5 \mathrm{~mm}$ de abertura. Uma lente convergente, extraída de uma lupa, foi fixada dentro da caixa de forma que seu foco $(f \approx 12 \mathrm{~cm})$ estivesse sobre a fenda de entrada. Para a obtenção dos espectros foi utilizada uma grade de difração comercial de 1000 fendas/mm e uma webcam (Logitech HD 720p, sem carcaça), as quais são mostradas na Figura 1(a). Na parte externa da caixa, foram usadas duas lentes convergentes $\left(\mathrm{L}_{1}\right.$ e $\left.\mathrm{L}_{2}\right)$ dispostas linearmente com focos na fonte luminosa e na fenda, de acordo com a Figura 1(b). 1

Para a obtenção de espectros de emissão de diodos emissores de luz (Light Emitting Diode-LED) foram usados LEDs comerciais e a medição da fotocorrente gerada pela excitação com diferentes comprimentos de onda foi feita por meio de um multímetro comercial e uma

\footnotetext{
1 Em termos do custo de produção, cada lupa foi adquirida por menos de $R \$ 4,00$, a caixa de MDF custou $R \$ 40,00$ e a webcam saiu por $\mathrm{R} \$ 120,00$. A grade de difração foi comprada em site de vendas dos Estados Unidos por aproximadamente $\mathrm{R} \$ 60,00$, sendo que a maior parte desse valou se deveu à taxa de entrega no Brasil. No entanto, é possível substituir este item por um CD/DVD. A base para o protótipo e as placas metálicas foram extraídas de sucata. A tinta usada custou menos de $\mathrm{R} \$ 10,00$, os parafusos aprox. $\mathrm{R} \$ 20,00$ e a lâmpada halógena, também $\mathrm{R} \$ 20,00$. Assim, o protótipo foi confeccionado por aproximadamente $\mathrm{R} \$ 282,00$. Valores referentes a $2017 / 2018$
}
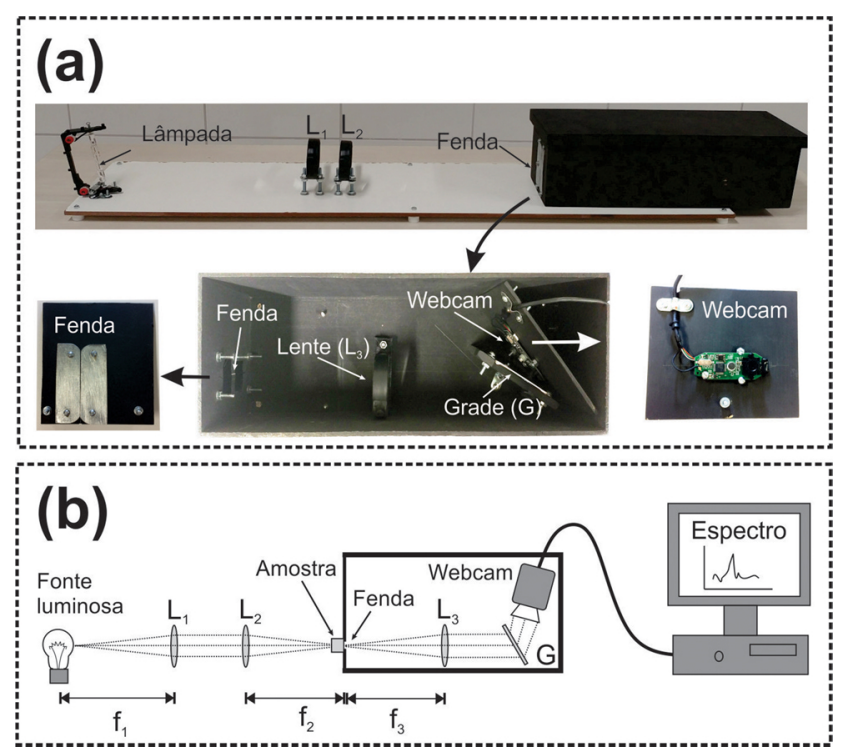

Figura 1: (a) Imagem do protótipo construído mostrando lâmpada halógena, lentes convergentes, fenda e caixa do espectrômetro, a qual tem sua parte interna mostrada em detalhes na parte inferior da figura. (b) Esquema mostrado caminho óptico no protótipo construído. Para a obtenção de espectros de emissão de fontes luminosas, a posição assinalada para a amostra permanece não ocupada, com o feixe luminoso passando livremente pela fenda.

fonte de tensão de corrente contínua (CC). Para a obtenção do espectro de absorção, utilizou-se como fonte luminosa uma lâmpada halógena alimentada por duas fontes de tesão CC associadas em série. A obtenção dos espectros a partir das imagens captadas pela webcam foi realizada usando o software Tracker [4]. Para exemplificar a aplicação do protótipo construído em relação à obtenção de um espectro de absorção, utilizou-se como amostra extrato de beterraba vermelha de mesa (Beta vulgaris L.). Para a retirada do extrato, uma pequena porção de beterraba foi macerada em um almofariz com a adição de álcool etílico hidratado $\left(46^{\circ}\right)$. Por fim, a mistura foi filtrada usando filtro de papel. Para a obtenção dos espectros de absorção, imagens de absorção do porta-amostras (cubeta) com álcool hidratado (solvente) foram obtidas usando como fonte de radiação a lâmpada halógena. Em seguida, os espectros de absorção dos extratos (os quais possuem álcool) foram coletados seguindo a mesma metodologia. A posição em que a amostra foi colocada está indicada na Figura 1(b). O software Tracker foi usado para obter os espectros individuais. A validação dos resultados obtidos foi feita através de um espectrômetro comercial da marca Nova Instruments®. 


\section{Resultados e Discussão}

\subsection{A Física Quântica e a aplicabilidade da espectroscopia óptica em sala de aula}

A introdução de ideias relativas à Física Moderna, em especial à Teoria Quântica, vem sendo bastante discutida na literatura $[5,6]$. Dentre as principais motivações apontadas, é central a necessidade de conceitos de Física Quântica para o entendimento sobre os aspectos básicos relacionados ao mundo tecnológico em que vivemos. Um problema apresentado em relação ao ensino desta área da Física é que esta é considerada, além de conceitualmente difícil, abstrata [6]. Como resultado, pode-se ter a impressão de que ela não traduz de forma apropriada a realidade. Nesse sentido, o uso da espectroscopia óptica pode trazer ganhos metodológicos importantes por apresentar os conceitos básicos de Física Quântica para explicar os resultados encontrados via análise espectral de fontes de luz de uso corriqueiro, bem como explorar a Física envolvida na obtenção e análise de espectros de absorção óptica.

De forma geral, a confecção de um espectrômetro e sua utilização para o estudo dos espectros luminosos de lâmpadas florescentes e incandescentes, LEDs ( Light Emitting Diodes), lasers, etc. permite tratar conceitos de Física Quântica relacionados à interação entre radiação e matéria. Átomos, íons e moléculas podem existir somente em estados discretos de energia. A mudança de um estado para outro (chamada de transição) está associada com a emissão e a absorção de um fóton. O comprimento de onda da radiação emitida ou absorvida é dado por:

$$
E_{2}-E_{1}=h \nu_{21}
$$

sendo que $E_{1}$ e $E_{2}$ representam os níveis de energia discretos dos estados envolvidos na transição, $\nu_{21}$ é a frequência da radiação emitida/absorvida e $h$ é a constante de Planck $\left(h=6,63 \times 10^{-34} \mathrm{~J} . s\right)$ [7]. Como iremos tratar, estes conceitos são essenciais para o entendimento dos fenômenos relacionados com as diferentes fontes luminosas e podem ser explorados em sala de aula.

\subsection{Calibração do espectrômetro didático e vantagens do uso do software Tracker}

Antes de mais nada, para o uso efetivo do espectrômetro didático é necessário realizar sua calibração. É possível executar esta etapa através da equação para grades de difração, $n \lambda=d \sin \theta$, em que $\lambda$ é o comprimento de onda, $d$ é a distância entre fendas adjacentes e $n=1,2,3, \ldots$ Uma vez que o feixe de luz incide sobre a grade de difração em um ângulo $\theta_{i} \neq 0^{\circ}$, essa equação precisa ser modificada para: $n \lambda=d\left(\sin \theta_{D}+\sin \theta_{i}\right)$, sendo $\theta_{D}$ o ângulo de difração (veja Figura 2). Portanto, conhecendo-se o valor de $d$, medindo-se $\theta_{i}$ diretamente, $\lambda$ seria função de $\theta_{D}$. Uma forma simples de se determinar esse ângulo é através da inserção de uma escala graduada em frente

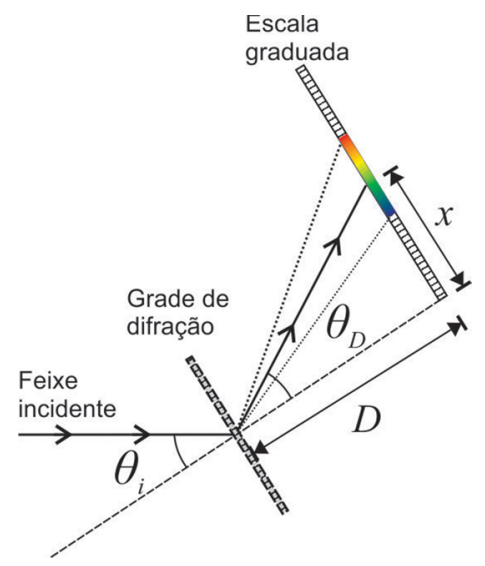

Figura 2: Esquema mostrando a geometria de uma das possíveis formas de calibração do espectrômetro, a qual é feita pela inserção de uma escala graduada onde o espectro de uma determinada fonte de luz seria projetado. Na figura, $\theta_{i}$ é o ângulo de incidência do feixe luminoso, $\theta_{D}$ é o ângulo de difração e $x$ é a posição em que determinada parte do espectro é projetada na escala.

à câmera, na qual o espectro pode ser projetado. Considerando que determinada parte do espectro é projetada na posição $x$ da escala, o valor de $\lambda$ correspondente pode ser obtido por:

$$
\lambda=\frac{d}{n}\left(\frac{x}{\sqrt{D^{2}+x^{2}}}+\sin \theta_{i}\right)
$$

Dessa forma, determina-se o comprimento de onda de partes características do espectro projetadas na escala graduada, as quais podem ser usadas para calibração do espectrômetro quando o mesmo espectro for captado pela webcam.

No entanto, uma maneira muito mais prática e menos susceptível a erros para se realizar a calibração é pela obtenção de um espectro de emissão de uma fonte de radiação conhecida, isto é, pela coleta de um espectro com características reconhecíveis e que se manifestam em comprimentos de onda determinados. Dentre as opções disponíveis, o espectro de uma lâmpada fluorescente comercial é o mais acessível. Assim, a calibração do dispositivo pode ser feita simplesmente a partir de um espectro de emissão de uma lâmpada fluorescente, cujas propriedades principais são descritas a seguir. É importante mencionar que neste caso torna-se desnecessário conhecer o valor de $d$, o que demandaria uma etapa adicional caso se utilizasse um CD/DVD (Compact Disc/Digital Video Disc) como grade de difração. Na referência [8] demonstra-se como $d$ poderia ser encontrado caso um CD/DVD fosse usado.

A Figura 3 mostra uma figura de difração obtida de uma lâmpada fluorescente compacta usando o espectrômetro construído. Uma lâmpada fluorescente é um objeto de uso corriqueiro, porém extremamente rico sob o ponto de vista da Física. Em uma lâmpada de descarga elétrica, como é o caso da lâmpada fluorescente, a luz é produ- 


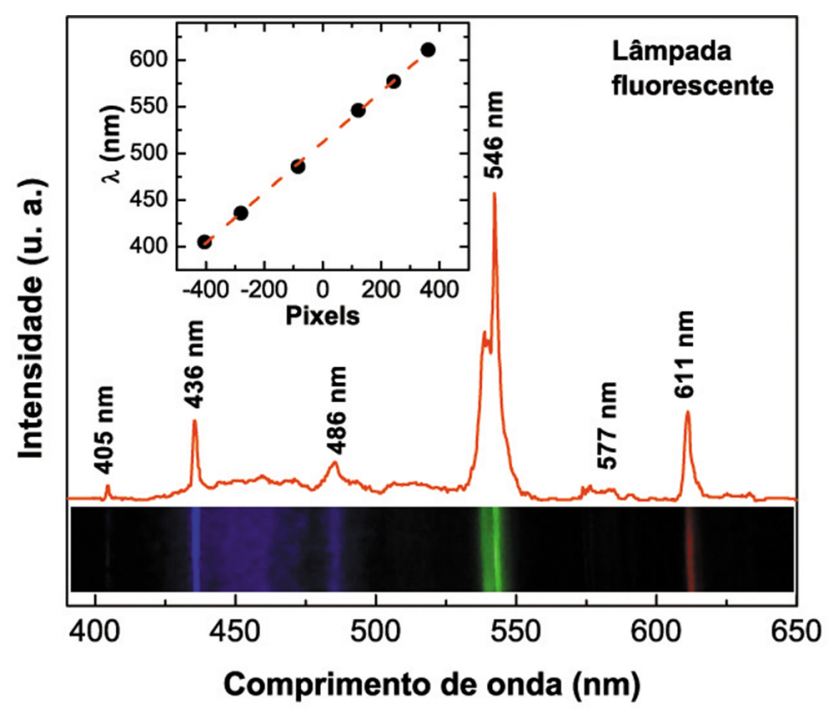

Figura 3: Espectro de emissão de lâmpada fluorescente obtido com o software Tracker a partir da imagem espectral na parte inferior da figura. Relação entre comprimento de onda $\lambda$ e pixels para a lâmpada fluorescente.

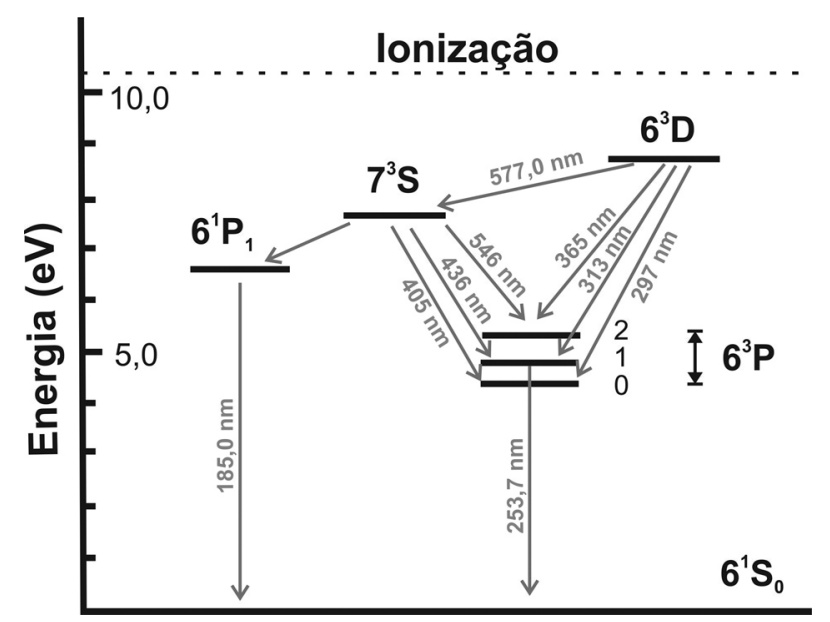

Figura 4: Transições eletrônicas possíveis em um átomo de $\mathrm{Hg}$ [9]. Linhas pretas horizontais representam os diferentes estados de energia disponíveis no mercúrio. Valores em nm (nanômetros) posicionados sobre as setas em cinza informam o comprimento de onda da luz emitida em cada uma das transições.

zida em três passos: um campo elétrico acelera elétrons; esses elétrons colidem com átomos gasosos ou moléculas, excitando-os para níveis de energia acima do estado fundamental; finalmente, os estados excitados decaem para estados de menor energia com a emissão de radiação [9]. A Figura 4 mostra um diagrama de níveis de energia do mercúrio $(\mathrm{Hg})$, o qual é usado na forma de vapor em uma lâmpada fluorescente. No $\mathrm{Hg}$, existem quatro transições eletrônicas $\left({ }^{3} \mathrm{P}_{0} \rightarrow 7^{3} \mathrm{~S},{ }^{3} \mathrm{P}_{1} \rightarrow 7^{3} \mathrm{~S},{ }^{3} \mathrm{P}_{2} \rightarrow\right.$ $7^{3} \mathrm{~S}$ e $\left.7^{3} \mathrm{~S} \rightarrow{ }^{3} \mathrm{D}\right)$ que são responsáveis pelas emissões com $\lambda=405 \mathrm{~nm}, 436 \mathrm{~nm}, 546 \mathrm{~nm}$ e $577 \mathrm{~nm}$ detectadas na imagem mostrada. A emissão com $\lambda=611$ nm não se deve ao mercúrio, mas sim a uma transição de dipolo elétrico $\left({ }^{5} \mathrm{D}_{0} \rightarrow{ }^{7} \mathrm{~F}_{2}\right)$ do íon európio no $\mathrm{Eu}^{3+}: \mathrm{Y}_{2} \mathrm{O}_{3}$, o qual é um material componente da camada fosfórica da lâmpada. Para essa emissão, o $\mathrm{Eu}^{3+}: \mathrm{Y}_{2} \mathrm{O}_{3}$ absorve fótons com $\lambda=254 \mathrm{~nm}$ (do mercúrio) através de uma transição de transferência de carga envolvendo o íon $\mathrm{Eu}^{3+}$ e um íon $\mathrm{O}^{2-}$ vizinho [10]. O pico de emissão em $486 \mathrm{~nm}$, por outro lado, pode ser atribuído a uma transição eletrônica $\left({ }^{5} \mathrm{D}_{4} \rightarrow{ }^{7} \mathrm{~F}_{6}\right)$ associada aos íons de térbio $\left(\mathrm{Tb}^{3+}\right)$ no $\mathrm{Ce}^{3+}, \mathrm{Tb}^{3+}: \mathrm{LaPO}_{4}$, o qual também compõem a camada fosfórica da lâmpada [11]. Outras linhas de emissão de menor intensidade também podem observadas em espectros de lâmpadas fluorescentes. Estes picos, da mesma forma, podem ser atribuídos aos íons $\mathrm{Tb}^{3+}[12]$. A Tabela 1 resume estas informações.

Para a calibração do protótipo, bem como a obtenção dos espectros (emissão e absorção) apresentados neste trabalho, utilizou-se o software Tracker. Este software é destinado à videoanálise e à modelagem, baseando-se em uma plataforma em Java. O Tracker é uma ferramenta bastante explorada para aplicações em ensino de Mecânica, porém, apresenta recursos úteis para a área de Óptica [13]. No Tracker, é possível obter o perfil de brilho ao longo de determinada direção em uma imagem digital (ver Figura 5). Além disso, o espectro obtido pode ser salvo em formato de tabela de dados (.txt, .dat, .xls, etc.), o que permite o tratamento posterior dos espectros em um software de análise gráfica. Desde que se tenha alguma referência é possível calibrar o software e obter espectros com boa qualidade. Dessa forma, os picos de emissão em $405 \mathrm{~nm}, 436 \mathrm{~nm}, 486 \mathrm{~nm}, 546 \mathrm{~nm}$, $577 \mathrm{~nm}$ e $611 \mathrm{~nm}$ podem ser usados para a calibração do espectrômetro/software. O quadro da Figura 3 mostra a posição na imagem (em pixels) em que os picos listados anteriormente se manifestam. Como é possível observar, a relação entre $\lambda$ e pixels é satisfatoriamente linear, mostrando que não ocorrem deformações da imagem. Tomando-se o cuidado de não alterar as posições relativas das partes do espectrômetro (principalmente grade de difração e câmera), imagens obtidas de outras fontes luminosas seguirão a reta experimental mostrada no quadro da Figura 3. No material suplementar deste artigo existem detalhes adicionais sobre o processo de calibração do espectrômetro.

Na literatura existem trabalhos mostrando a confecção de espectrômetros didáticos baseados em webcam. Muitos destes usam o software Tracker para a extração

Tabela 1: Principais transições eletrônicas associadas às linhas de emissão observadas no espectro visível de uma lâmpada fluorescente compacta.

\begin{tabular}{lcc}
\hline$\lambda(\mathrm{nm})$ & Transição & Origem \\
\hline 405 & ${ }^{3} \mathrm{P}_{0} \rightarrow 7^{3} \mathrm{~S}$ & $\mathrm{Hg}$ \\
\hline 436 & ${ }^{3} \mathrm{P}_{1} \rightarrow 7^{3} \mathrm{~S}$ & $\mathrm{Hg}$ \\
\hline 486 & ${ }^{5} \mathrm{D}_{4} \rightarrow{ }^{7} \mathrm{~F}_{6}$ & $\mathrm{~Tb}$ \\
\hline 546 & ${ }^{3} \mathrm{P}_{2} \rightarrow 7^{3} \mathrm{~S}$ & $\mathrm{Hg}$ \\
\hline 577 & $7^{3} \mathrm{~S} \rightarrow{ }^{3} \mathrm{D}$ & $\mathrm{Hg}$ \\
\hline 611 & ${ }^{5} \mathrm{D}_{0} \rightarrow{ }^{7} \mathrm{~F}_{2}$ & $\mathrm{Eu}$ \\
\hline
\end{tabular}




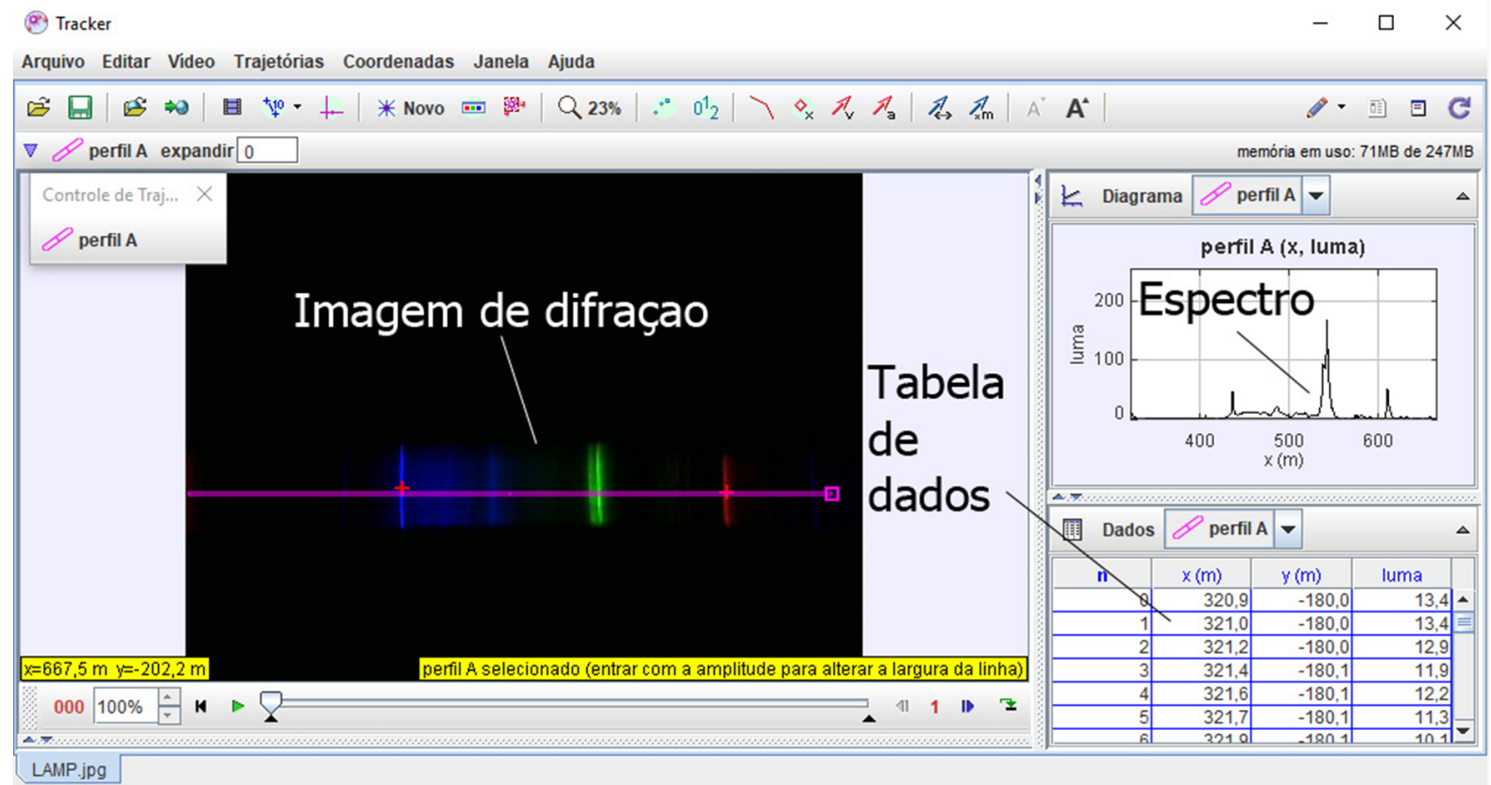

Figura 5: Tela do software Tracker mostrando a extração de espectro a partir de imagem de difração. O espectro extraído da figura e a respectiva tabela de dados são mostrados à direita na figura.

dos espectros a partir de uma imagem de difração. Em Onorato et al. [14], mostra-se a confecção e o emprego de um espectrômetro de baixo custo para estudar o espectro fluorescente de tintas coloridas de canetas (marcadoras). Além disso, existe exemplo de aplicação de espectroscopia em ensino em que se utiliza o software Tracker em complemento à tecnologia Arduino [15]. Também é possível encontrar exemplos da confecção do mesmo tipo de dispositivo para a caracterização de nanomateriais [13]. Além do Tracker, existem outros softwares para obtenção de espectros de difração via webcam. Uma dessas possiblidades é o software ImageJ [16, 17], o qual também possui código aberto e é bastante usado para uma série de tarefas científicas, como determinação do tamanho médio de nanopartículas [18], análise de tecido biológicos, neurociência, etc. [19]. Também é reportada a utilização dos softwares Labview [20], RSpec [3], e MATLAB [21]. Para aquisição de espectros ópticos instantâneos, excelentes para demonstrações rápidas, existe a opção de instalar o software livre Theremino Spectrometer [22]. No entanto, tal aplicativo não permite salvar dados em forma de tabela, de modo que não possibilita, por exemplo, a obtenção de espectros de absorção, como mostrado mais adiante. Para esta finalidade, o uso do Tracker é mais indicado. Informações adicionais sobre o uso do Tracker estão disponíveis no material suplementar deste artigo.

\subsection{Aplicações didáticas de espectros de lâmpadas, LEDs e lasers}

Em relação ao espectro de emissão da lâmpada fluorescente, além da possibilidade de sua utilização para a calibração do dispositivo, é possível explorar as propriedades de seu espectro. A principal característica que

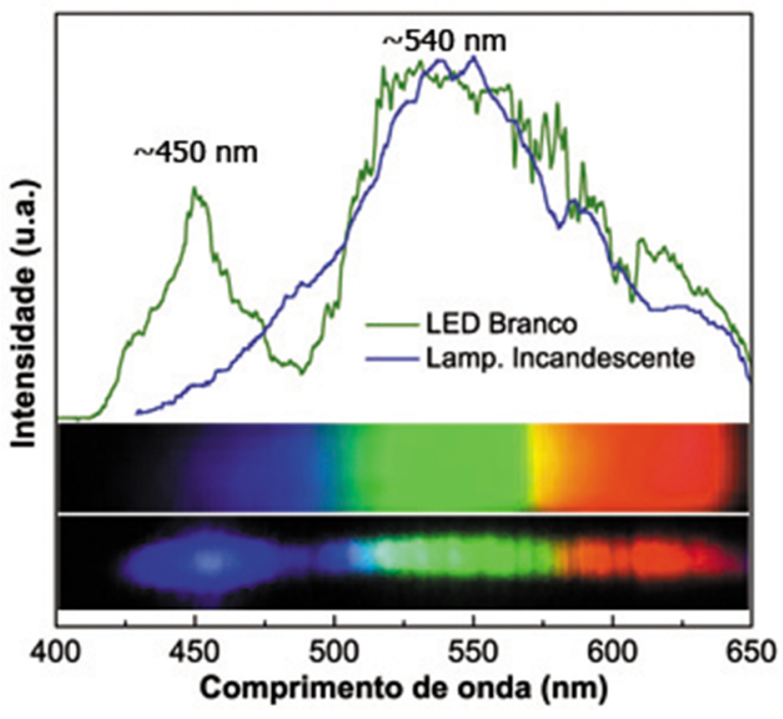

Figura 6: Imagens espectrais (parte inferior da figura) e espectros (parte superior) de lâmpada incandescente e LED branco.

pode ser mostrada pelo espectro da lâmpada fluorescente (Figura 3) é seu carácter descontínuo. Diferentemente do que é possível observar dos espectros da lâmpada halógena e do LED branco (Figura 6), nem todas as cores (comprimentos de onda) estão presentes na imagem da Figura 3. Dessa forma, a utilização do espectrômetro didático permite ao professor demonstrar uma das evidências experimentais mais expressivas que levaram ao surgimento da hipótese quântica: a verificação de espectros de gases com emissões descontínuas. Além disso, pode-se explorar conceitos de composições de cores e o fato de que espectralmente as três fontes luminosas de cor 
branca possuem espectros distintos. Cabem aqui algumas reflexões. Primeiro, a complexidade dos níveis de energia do mercúrio (Figura 4) é secundária em relação ao fato de que experimentalmente se obtém evidências de níveis de energia discretos. Segundo, já era conhecido no fim do Século XIX que átomos podiam emitir (e absorver) luz em apenas certos comprimentos de onda bem definidos. Esses experimentos, como se sabe, deram a base para as teorias atômicas desenvolvidas no Século XX [23]. Apesar dos experimentos mais importantes terem sido realizados com gás hidrogênio [24], a abordagem em sala de aula baseando-se no espectro da lâmpada fluorescente (majoritariamente nas linhas de emissão do $\mathrm{Hg}$ ) é capaz de ilustrar suficientemente os resultados experimentais observados naquela época. Finalmente, os processos de excitação dos íons terras-raras, os quais compõe a superfície fosfórica da lâmpada, podem exemplificar como se dá o processo de excitação eletrônica e emissão de radiação.

Outra possibilidade para a introdução de temas relacionados à Física Moderna na educação básica via espectroscopia pode ser através do uso de diodos emissores de luz (Light Emitting Diodes-LED) e de lasers (Light Amplification by Stimulated Emission of Radiation). A Figura 7 mostra os espectros de emissão de LED

(a)
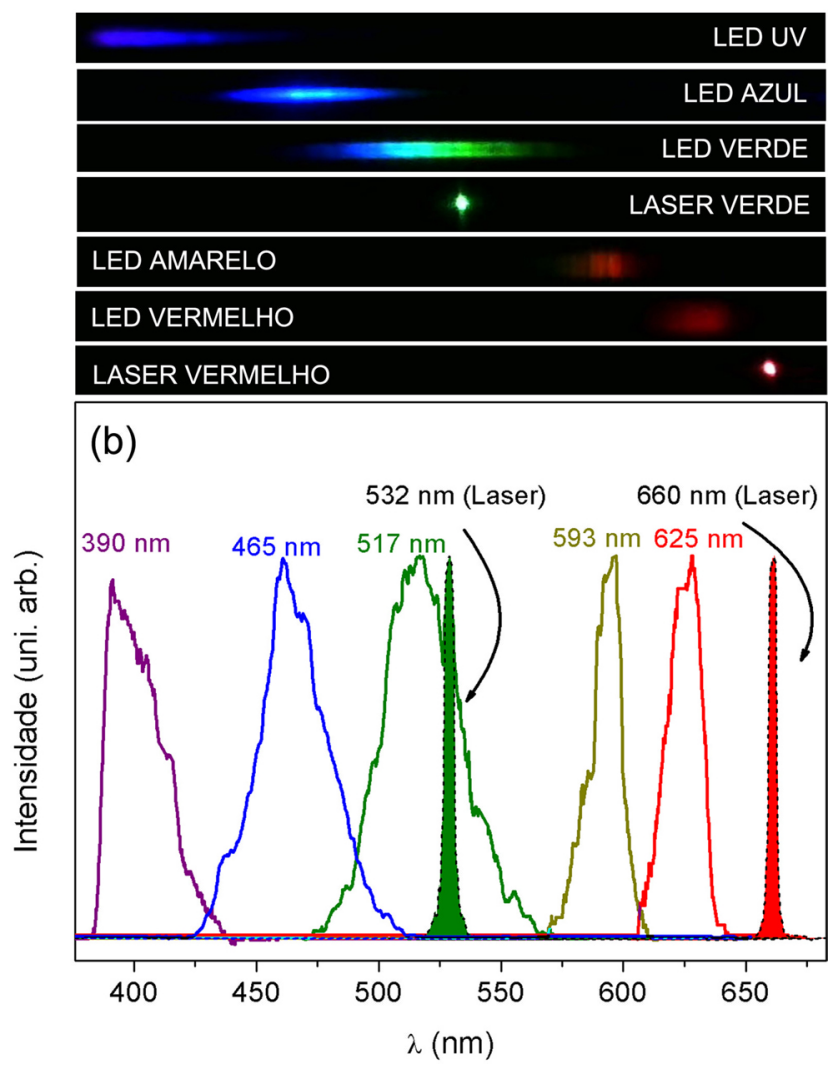

Figura 7: Imagens espectrais (a) e espectros de emissão (b) de LEDs ultravioleta, azul, verde, amarelo e vermelho, bem como espectros de emissão de lasers verde e vermelho. ultravioleta (UV), azul, verde, amarelo e vermelho. Além disso, a Figura 7 também mostra os espectros de emissão de dois lasers (tipo pointers), um verde e um vermelho. Para efeito de comparação, a intensidade dos picos foi normalizada. LEDs são diodos que emitem luz com o fluxo de corrente elétrica [25]. Como uma primeira aproximação, LEDs consistem em um material semicondutor dopado de forma controlada com impurezas com o intuito de criar uma junção $p-n$. Nesses materiais, portadores de carga (elétrons e lacunas) ao fluírem pela junção se recombinam, decaindo para um nível de mais baixa energia ao emitir um fóton. O comprimento de onda da luz emitida, e, portanto, sua cor, depende da banda de energia proibida (band gap) do material [26]. No entanto, para se evitar a reabsorção dos fótons emitidos e, por consequência, a redução da eficiência do dispositivo, os LEDs modernos possuem uma região ativa, a qual possui energia de gap inferior à da parte tipo- $p$ e tipo- $n$ (Figura 8(a)). Assim, as energias dos fótons de luz medidas neste trabalho via espectroscopia óptica se referem às transições que ocorrem na região ativa. Dentre os materiais mais usados para a confecção de diodos emissores de luz, é possível destacar os semicondutores inorgânicos AlGaAs, AlGaP, AlGaInP, GaAsP, GaP, GaN, InGaN, etc. [27]. Para o LED branco, por outro lado, é presumese por seu espectro que este dispositivo é composto por dois materiais, um responsável pelo pico de emissão em $\sim 450 \mathrm{~nm}$ e outro pelo pico em $540 \mathrm{~nm}$. De fato, o pico de menor comprimento de onda provém, na maioria dos casos, de emissões do GaN ou InGaN, enquanto que o pico de maior $\lambda$ resulta de fótons da liga Ce: $\mathrm{Y}_{3} \mathrm{Al}_{5} \mathrm{O}_{12}$.

No caso do laser semicondutor, por outro lado, são necessários dois processos para seu funcionamento: o surgimento de uma inversão de população em uma junção $p-n$, isto é, da existência de maior número de elétrons em estados mais energéticos do que em estados de mais baixa energia, e um mecanismo para emissão estimulada. Para o surgimento de inversão de população, é preciso excitar o sistema através do fornecimento de energia de forma contínua, sendo que o modo mais simples é através de uma corrente elétrica suficientemente elevada. Para o processo de auto-estimulação, no entanto, emprega-se o fato de que quando um fóton é emitido devido à transição de um elétron da banda de condução (BC) para a banda de valência $(B V)$ este fóton pode estimular a transição de um segundo elétron para a BV, o qual poderá dar sequência ao processo e gerar um efeito cascata. Assim, empregando um cristal com faces opostas refletivas suficientemente planas e paralelas, de forma a favorecer o processo de emissão estimulada, é possível criar uma reação em cadeia que produz um feixe laser [28].

\subsection{Usando LEDs e lasers para explorar a relação entre a cor da luz e sua energia}

Além do efeito de eletroluminescência, para o qual um LED é desenvolvido, também é possível a ocorrência 
(a)

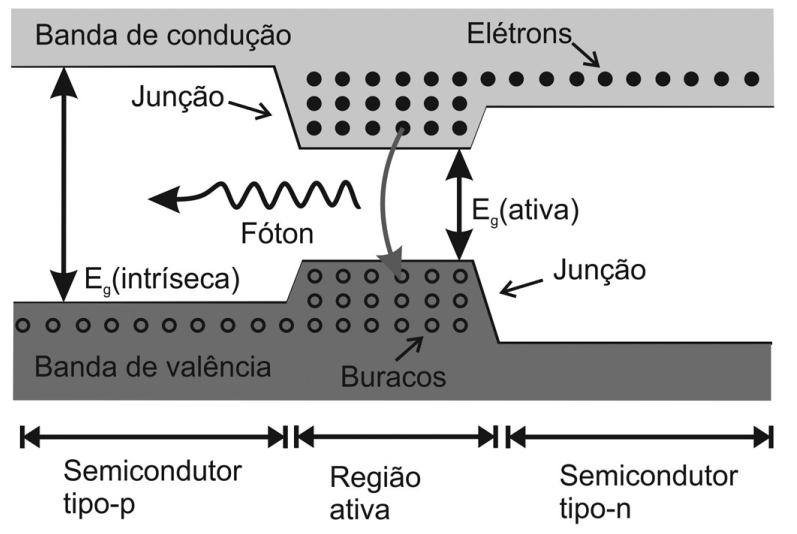

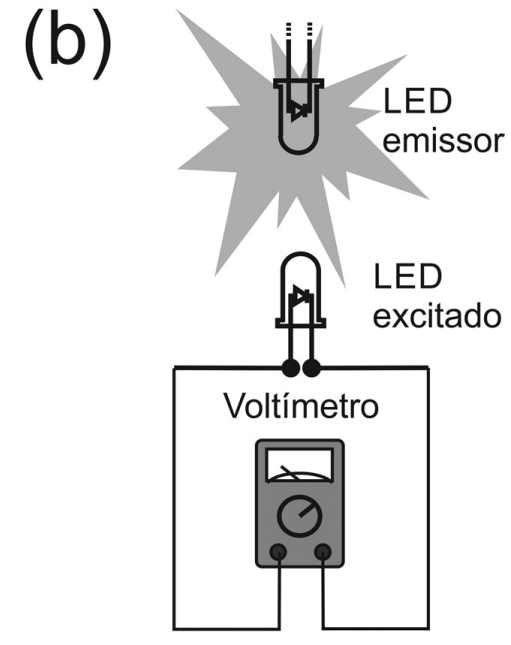

Figura 8: (a) Esquema mostrando a emissão em um LED (Adaptado de [27]). Faixa cinza mais escura representa a banda de valência do material e a faixa cinza mais claro a banda de condução. Pontos preenchidos representam elétrons e pontos não-preenchidos os buracos (ou lacunas). (b) Esquema da montagem experimental usada para a demonstração da dependência da fotocorrente nos LEDs com relação ao comprimento de onda da luz do LED (ou laser) emissor.
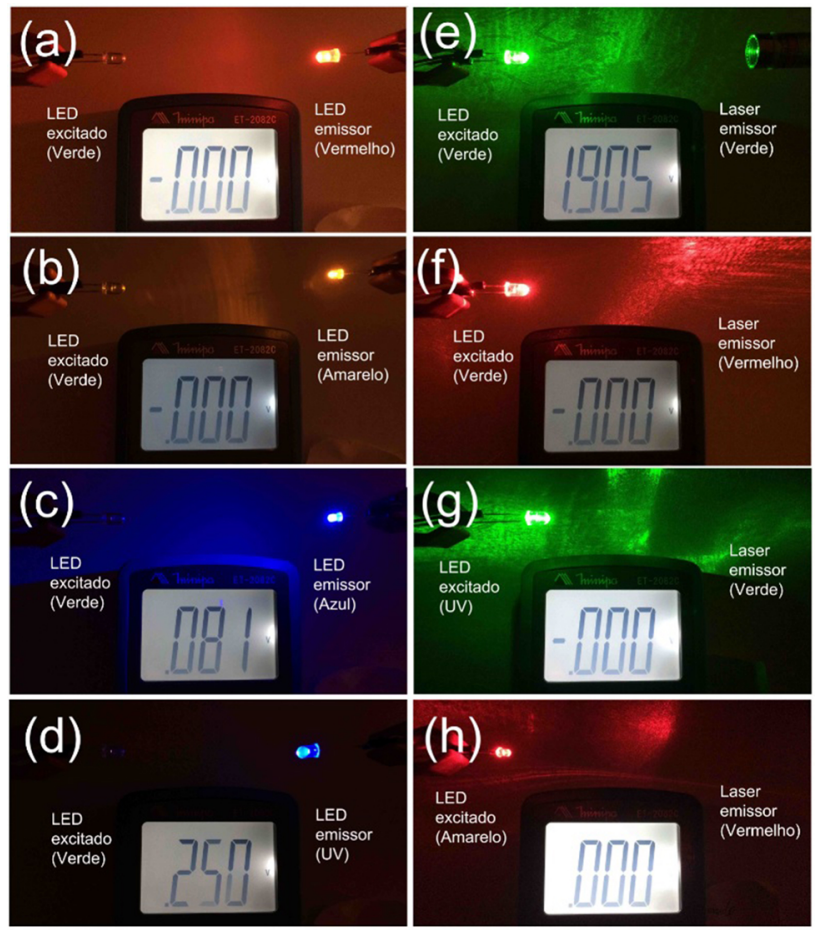

Figura 9: LED verde excitado com LED (a) vermelho, (b) amarelo, (c) azul e (d) ultravioleta. (e) Excitação de LED verde com laser (e) verde e (f) vermelho. Excitação do LED UV com laser verde e do LED amarelo com laser vermelho. A tela do multímetro mostrada nas imagens apresenta valores de tensão (CC) em volts.

de efeito inverso no dispositivo. Em outras palavras, a incidência de fótons de luz na superfície ativa de um LED pode gerar uma corrente elétrica. Este efeito pode ser explorado para a abordagem da Física Quântica em sala de aula. Na Figura 9, mostra-se a tensão gerada nos polos de diferentes LEDs pela excitação com diferentes valores de comprimento de onda $(\lambda)$, a qual é medida usando um multímetro, de acordo com o esquema da Figura 8(b). O intuito desta atividade é demonstrar que a excitação de fotocorrente nos LEDs depende da energia de excitação, bem como do band gap de energia do dispositivo usado. Assim, como é possível observar na Figura 9, a excitação do LED verde com luz do LED vermelho ou amarelo não leva ao aparecimento de tensão, como pode se ver pela marcação do voltímetro. Por outro lado, caso um LED azul (Figura 9(c)) ou um LED UV (Figura 9(d)) sejam usados como fonte de excitação, verifica-se o surgimento de uma diferença de potencial - DDP. Da mesma forma, apesar de não mostrado na Figura 9, é possível excitar fotocorrente em LED vermelho pela incidência de luz amarela, verde, azul e UV. Para o LED ultravioleta, no entanto, nenhum dos outros LEDs leva ao surgimento de DDP.

É importante observar nas atividades didáticas que a intensidade luminosa não justifica o aparecimento ou não de tensão nos polos do LED. Para isso, usamos dois lasers com potência nominal de aproximadamente $100 \mathrm{~mW}$, sendo um verde (de $\lambda \approx 532 \mathrm{~nm}$ ) e outro vermelho (de $\lambda \approx 660 \mathrm{~nm}$ ). Como é possível notar na Figura $9(\mathrm{e})$, a incidência direta da luz do laser verde sobre o LED verde leva ao aparecimento de tensão em seus terminais. O mesmo não ocorre para a excitação com o laser vermelho (Figura 9(f)). Caso o laser verde seja usado para excitar o LED UV, não se observa o surgimento de tensão nos terminais do LED (Figura 9(g)). O mesmo ocorre com a excitação via laser vermelho considerando, por exemplo, o LED amarelo (Figura 9(h)). Dessa forma, conclui-se que a produção de fotocorrente depende da cor da luz de excitação. Apesar de não mostrado na Figura 9, o laser vermelho permite gerar fotocorrente 
em LED infravermelho (IV) de $\lambda \approx 940 \mathrm{~nm}$. Usando os dados espectrais como aqueles mostrados na Figura 7 , os estudantes devem concluir que somente excitações com menor comprimento de onda (maior energia) são capazes de produzir fotocorrente nos terminais dos LEDs. O mesmo ocorre para a excitação via laser.

\subsection{Obtendo espectros de absorção óptica}

Outra possiblidade de exploração da espectroscopia óptica no contexto educacional é desenvolvida a seguir. A Figura 10 mostra, como exemplo de aplicação, o espectro de absorção obtido para o extrato de beterraba, usando o protótipo desenvolvido (linhas azuis) e um espectrômetro comercial (linhas vermelhas). Para o extrato de beterraba, observa-se que na região do visível, a absorbância decresce para valores de $\lambda$ superiores a $\sim 600 \mathrm{~nm}$, o que está de acordo com sua cor característica, a qual é determinada pela presença de alcaloides coloridos atóxicos e hidrossolúveis chamados de betalaínas [29] Como é possível perceber, a comparação entre as duas curvas demonstra boa concordância entre os resultados obtidos via protótipo e através do espectrômetro comercial. Considerando que para a produção do espectrômetro foram gastos menos de trezentos reais, tem-se que a confecção desse dispositivo apresenta excelente relação custo/benefício. É importante mencionar também que a escolha do tipo de substrato se deveu à facilidade de obtenção, simplicidade de extração e intensidade da cor apresentada. No entanto, as opções de aplicação do espectrômetro óptico se extendem a todas as substâncias coloridas de facil acesso a professores e estudantes.

Cabe destacar que a análise dos espectros ópticos obtidos permite mostrar que substâncias possuem assinaturas espectrais, as quais possibilitam sua caracterização e identificação. Além disso, existe a opção de estudar

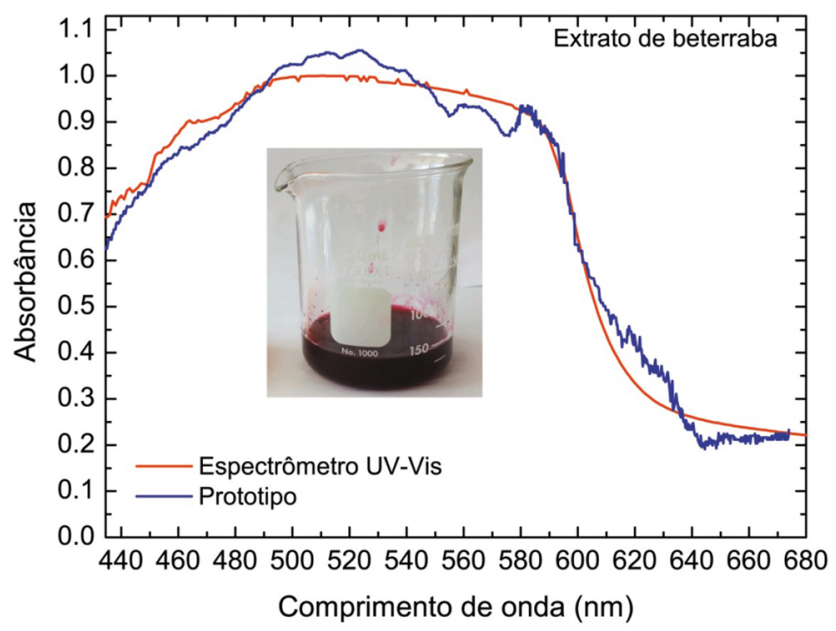

Figura 10: Espectro de absorção do extrato de beterraba. Linha azul é referente ao espectro obtido com o protótipo desenvolvido e a linha vermelha é o espectro obtido com espectrômetro UV-vis comercial. os conceitos de absorção de energia no contexto da interação entre radiação e matéria. Em especial, é possível quantificar esta relação através da lei de Beer-Lambert [30], a qual fornece a absorbância $(A)$ :

$$
A=-\log _{10}\left(\frac{I}{I_{0}}\right)
$$

em que $I_{0}$ é a intensidade total e $I$ é a intensidade absorvida pela amostra. Para tanto, a lei de Beer-Lambert é essencial para a montagem do espectro de absorção. Do software Tracker, após o processo de calibração descrito, é possível extrair uma tabela de dados com duas colunas, sendo que a primeira traz os valores de comprimento de onda e a segunda a intensidade (brilho). Assim, um espectro de absorbância pode ser obtido relacionando a intensidade medida sem a amostra, isto é, apenas com o porta-amostras (no nosso caso, uma cubeta de vidro) e o solvente (álcool etílico hidratado), e a intensidade medida quando a amostra (extrato de beterraba) é colocada no caminho óptico mostrado na Figura 1. Como o protótipo mede essencialmente a luz transmitida através de um material, é necessário subrair as intensidades obtidas com a amostra $\left(I_{\text {amostra }}\right)$ daquelas encontradas para a situação em que se utilizou apenas o porta-amostras e o solvente $\left(I_{0}\right)$. Dessa forma, isto é, fazendo $I=I_{0}-I_{\text {amostra }}$, obtem-se a intensidade absorvida. Assim, usados os valores de $I$ descritos anteriormente, a equação (3) e os respectivos valores de $\lambda$, é possível construir o gráfico mostrado na Figura 10.

\subsection{Parâmetros Curriculares Nacionais e o uso da espectroscopia na sala de aula}

A inserção da Física Moderna na educação básica e sua importância está descrita nos Parâmetros Curriculares Nacionais (PCN) para a área de Física, como se observa no trecho mostrado a seguir [31]:

A Ótica e o Eletromagnetismo, além de fornecerem elementos para uma leitura do mundo da informação e da comunicação, poderiam, numa conceituação ampla, envolvendo a codificação e o transporte da energia, ser o espaço adequado para a introdução e discussão de modelos microscópicos. A natureza ondulatória e quântica da luz e sua interação com os meios materiais, assim como os modelos de absorção e emissão de energia pelos átomos, são alguns exemplos que também abrem espaço para uma abordagem quântica da estrutura da matéria, em que possam ser modelados os semicondutores e outros dispositivos eletrônicos contemporâneos. (p. 26)

Nesse sentido, a utilização de um equipamento que congregue óptica e eletrônica, como é o caso do espectrômetro óptico, dá subsídios para a introdução de conteúdos relacionados à Físíca Quântica, de forma a favorecer o 
entendimento dos conceitos fundamentais associados à absorção e emissão de energia por átomos (modelos microscópios). Espectros obtidos com o protótipo construído deixam claras estas ideias, principalmente em relação ao espectro de emissão da lâmpada fluorescente. Considerando o estudo dos materiais semicondutores e dispositivos eletrônicos contemporâneos, como mencionado nos PCNs, nossos resultados mostram que a utilização do espectrômetro óptico em atividades didáticas cria um ambiente propício para a abordar os conceitos de bandas de energia, portadores de carga (elétrons e buracos), dopagem, transição eletrônica, fótons, etc. Em especial, o emprego de protótipo para a investigação da radiação absorvida/emitida por LEDs de diferentes cores pode ser usado em propostas didáticas de forma a evidenciar que a energia da radiação eletromagnética depende de sua cor (de $\lambda$, de uma forma um pouco mais formal). Além disso, fenômenos ondulatórios, como interferência, difração, refração, formação de imagens, etc. se fazem presentes durante a confecção e aplicação do espectrômetro. Considerando os processos de emissão/absorção, em que a explicação corpuscular é adotada, tem-se uma ótima oportunidade para se tratar da dualidade onda-partícula no contexto da espectroscopia.

Ainda em relação ao mesmo assunto, nas Orientações Educacionais Complementares aos Parâmetros Curriculares Nacionais $(\mathrm{PCN}+)[32]$, verifica-se:

\begin{abstract}
Alguns aspectos da chamada Física Moderna serão indispensáveis para permitir aos jovens adquirir uma compreensão mais abrangente sobre como se constitui a matéria, de forma a que tenham contato com diferentes e novos materiais, cristais líquidos e lasers presentes nos utensílios tecnológicos, ou com o desenvolvimento da eletrônica, dos circuitos integrados e dos microprocessadores. A compreensão dos modelos para a constituição da matéria deve, ainda, incluir as interações no núcleo dos átomos e os modelos que a ciência hoje propõe para um mundo povoado de partículas. Mas será também indispensável ir mais além, aprendendo a identificar, lidar e reconhecer as radiações e seus diferentes usos. Ou seja, o estudo da matéria e radiação indica um tema capaz de organizar as competências relacionadas à compreensão do mundo material microscópico. (p. 19)
\end{abstract}

Em relação às potencialidades associadas à aplicação da espectroscopia no contexto da educação básica, é natural, por exemplo, a investigação da radiação produzida por lasers, bem como a discussão sobre os fenômenos associados à sua produção. Neste caso, uma análise espectroscópica da luz emitida por estes dispositivos torna-se fundamental. Em relação ao tema das radiações e seus diferentes usos, como mencionado nos $\mathrm{PCN}+$, o protótipo desenvolvido traz amplas possibilidades em relação a luz visível, IV e UV. A investigação sobre os espectros de absorção de algumas substâncias, da mesma forma, pode ser empregada no contexto do ensino da interação radiação-matéria, além de exemplificar as potencialidades investigativas da espectroscopia dentro da ciência de materiais.

\section{Conclusões}

A confecção de um kit de espectroscopia óptica de baixo custo possui grande potencial para a introdução de conceitos e ideias de Física Quântica no âmbito da educação básica. Em especial, a obtenção de espectros de emissão de lâmpadas fluorescentes, LEDs e lasers dá subsídios concretos para se discutir conceitos relacionados com quantização de energia, transições eletrônicas, bandas de energia, emissão e absorção de fótons, etc. Da mesma forma, experimentos simples envolvendo a fotoexcitação de LEDs podem ser utilizados para demonstrar que a energia de um fóton depende de seu comprimento de onda. Por outro lado, também é possível empregar o protótipo construído para a obtenção de espectros de absorção. Os dados apresentados neste artigo mostraram boa concordância quando comparados com aqueles encontrados usando um espectrômetro UV-vis comercial. Isso mostra que medidas de absorbância podem ser usadas para abordar fenômenos relacionados com a interação entre radiaçao e matéria, bem como para ilustrar o papel que as técnicas espectroscópicas desenpenham no estudos de novos materiais. Assim, conclui-se que a confecção de um espectrômetro de baixo custo e sua inserção em um ambiente de ensino possui grandes potencialidades para o ensino de Física, em especial para a área de Física Quântica.

\section{AGRADECIMENTOS}

Os autores agradecem: à Pró-Reitoria de Pesquisa e Inovação do Instituto Federal de Brasília (PRPI-IFB) pela conceção de bolsa e auxílio financeiro para o desenvolvimento do projeto apresentado neste artigo; aos avaliadores pela leitura atenta e valiosas sugestões.

\section{Material Suplementar}

Os seguintes materiais suplementares estão disponíveis online:

Guia para calibração e uso do software Tracker.

\section{Referências}

[1] J.G. Solé, L.E. Bausá e D. Jaque, An introduction to Optical Spectroscopy of Inorganic Solids (John Wiley \& Sons, Chichester, 2005).

[2] D.O. Leite e R. J. Prado, Rev. Bras. Ensino Fís. 34, 2504 (2012). 
[3] J.R. Vanderveen, B. Martin e K.J. Ooms, J. of Chem. Educ. 90, 894 (2013).

[4] Tracker, acessado em 20/03/2018: disponível em https://physlets.org/tracker/.

[5] F. Ostermann e M.A. Moreira, Investigações em Ensino de Ciências 5, 23 (2000).

[6] E.C. Valadares e A.M. Moreira, Cad. Cat. Ens. Fis. 15, 121 (1998)

[7] W. Koechner, Solid-State Laser Engineering (Springer, New York, 2006), $6^{\text {a }}$ ed.

[8] H. Kruglak, Phys. Educ. 26, 255 (1991).

[9] H.S.W. Massey, E.W. McDaniel e B. Bederson, Applied Atomic Collision Physics (Academic Press, New York, 1982).

[10] A.M. Srivastava e T.J. Sommerer, Electrochemical Society Interface 7, 28 (1998).

[11] F.S. Kao e T.M. Chen, J. Lumin. 96, 261 (2002).

[12] B. Moine e G. Bizarri, Mater. Sci. Eng. B105, 2 (2003).

[13] A.Y. Nuryantine, E.C.S. Mehen, A. Sawitri e B.W. Nuryadin, Eur. J. Phys. 38, 055501 (2017).

[14] P. Onorato, M. Magieri e A. de Ambrosis, Eur. J. Phys. 37, 015301 (2016).

[15] M.A. Cavalcante, A.C. Teixeira e M. Balaton, Física na Escola 14, 27 (2016).

[16] ImageJ software, acessado em 21/10/2018: disponível em https://imagej.nih.gov/ij/.

[17] R.D. Lorenz, Am. J. Phys. 82, 169 (2014).

[18] T.J. Collins, Biotechniques, 43, 25 (2007).

[19] M.D. Abràmoff, P.J. Magalhães e S.J. Ram, Biophotonics Int. 11, 36 (2004).

[20] Z. Qu, J. Engstrom, D. Wong, M. Islam e C.F. Kaminski, Analyst 138, 6372 (2013).

[21] O. Ormachea e O. Urquidi e J. Cisneros, Rev. Cub. Fis. 30, 93 (2013).

[22] Theremino Software, acessado em 21/10/2018: https://www.theremino.com/en/downloads.

[23] O. Sala, Quim. Nova, 30, 1773 (2007).

[24] J.M.F. Bassalo e R.F. Farias, Rev. Bras. Ensino Quim. 9, 93 (2014)

[25] M.H. Chang, D. Das, P.V. Varde e M. Pecht, Microelectron. Reliab. 52, 756 (2012).

[26] S.C. Singh, in Handbook of Light Emitting and Schottky Diode Research, editado por N.P. Chen (Nova Scientific Publishers, New York, 2009).

[27] E.P. Wagner, J. Chem. Educ. 93, 1289 (2016).

[28] T. Suhara, Semiconductor Laser Fundamentals (Marcel Dekker, New York, 2004), $1^{\mathrm{a}}$ ed.

[29] L.C.P. Gonçalves, A.C. Marcato, A.C.B. Rodrigues, A.P.E. Pagano, B.C. Freitas, C.O. Machado, K.K. Nakashima, L.C. Esteves, N.B. Lopes e E. L. Bastos, Rev. Virtual Quim. 7, 292 (2015).

[30] A.F. Moreira, S.R.B. Santos e A.G. Costa Junior, Holos 2, 142 (2016).

[31] BRASIL, Parâmetros Curriculares Nacionais: ensino médio (Ministério da Educação, Brasília, 1999).

[32] BRASIL, PCN+ Ensino Médio: orientaçôes educacionais complementares aos Parâmetros Curriculares Nacionais (Ministério da Educação, Brasília, 2002). 